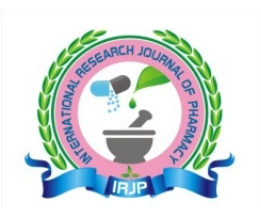

INTERNATIONAL RESEARCH JOURNAL OF PHARMACY

www.irjponline.com

ISSN $2230-8407$

\title{
Research Article \\ IDENTIFYING DRUG-DRUG INTERACTION AMONG CHRONIC KIDNEY DISEASE PATIENTS IN TERTIARY CARE HOSPITAL
}

Yasaman Ghanbari, Kiran Nagaraju *

Department of Pharmacy Practice, Kempedowda Institute of Medical Science Hospital and Research Centre, V.V Puram, Bangalore, Karnataka, India

*Corresponding Author Email: kirantoxicology@gmail.com

Article Received on: 21/05/16 Revised on: 30/06/16 Approved for publication: 18/07/16

DOI: $10.7897 / 2230-8407.07785$

\section{ABSTRACT}

Drug-drug interactions (DDIs) are common in clinical practice and are directly related to factors such as polypharmacy, aging, hepatic metabolism and decreased renal function. Individuals with chronic kidney disease (CKD) often require multiple classes of drugs being at important risk for the development of DDIs. The objectives of this study were to identify the drug-drug interactions in CKD patients admitted to medicine ward in KIMS Hospital \& Research Centre, and to categorize the drug-drug interactions based on their severity. The prescriptions of 108 patients who fulfilled inclusion criteria of study were analyzed during the study period to determine drug-drug interactions (DDIs). Out of total patient included in the study, in 78 patients medical record, 388 DDIs has been found during patient's medical record analysis. On correlating between patient`s age group and DDIs it has been found that most number of recorded DDIs, 33 patients $(30.56 \%)$ were belong to age group between $51-70$ years. DDIs has been classified into serious, significant and minor. Out of total 388 DDIs, 57 (14.96\%), 220 (56.7\%) and 111 (28.61\%) were serious, significant and minor DDIs respectively. Among all prescribed drugs, diuretics were among drugs with highest number of DDIs followed by antihypertensive agents, hypoglycemia agents and antithrombotic agents respectively. In relationship between polypharmacy and DDIs the highest number of patients, 48 (44.44\%) have been received 13-17 medications. Patients with renal insufficiency are at high risk of drug-drug interactions, especially in the later stages of the disease. Monitoring patient's medication chart by clinical pharmacist to detect DDIs early can improve quality of patient health care and provide appropriate mechanism for management of drug therapy among CKD patients.

Keywords: Chronic kidney disease, drug-drug interaction.

\section{INTRODUCTION}

Chronic kidney disease (CKD) represents a major public health problem in developed and developing countries, which is defined as serum creatinine concentrations greater than 1.2 to $1.5 \mathrm{mg} / \mathrm{dL} .{ }^{1}$ The kidneys play a key role in the maintenance of homeostasis and in regulatory, excretory and endocrine functions. Therefore, the gradual decreases in the glomerular filtration rate (GFR) and/or loss of kidney function seen in CKD patients compromise the homeostasis of the entire body. ${ }^{2}$ Patients with CKD often have alterations in pharmacokinetic parameters such as drug absorption, distribution, protein binding, metabolism and renal excretion. Many medications and their metabolites are eliminated by the kidney and thus adequate renal function is important to avoid toxicity. Patients with severe renal insufficiency can experience an accumulation of metabolites which can contribute to pharmacological activity or toxicity. Patients can also have an altered pharmacodynamic response to a given drug due to physiological and biochemical changes associated with progressive renal insufficiency. ${ }^{3,4}$ $\mathrm{CKD}$ and end-stage renal disease (ESRD) are associated with an increased risk of mortality, increased rate of hospitalization, and decreased life expectancy. ESRD patients who are on hemodialysis have complex drug regimens and receive nearly 10 to 12 medications daily, many of which requires multiple doses/day. Due to polypharmacy, frequent medication adjustments on dialysis versus non-dialysis days, medically unstable nature of the disease and restricted life styles, these patients are at high risk for drug-drug interactions (DDIs). ${ }^{5} \mathrm{In}$ clinical practice, multiple drugs are often combined in the treatment of patients with chronic diseases. These associations generally produce DDIswith expected beneficial effects, but in some cases undesired outcomes may also occur, such as ineffective treatment and severe adverse events.

DDIs can be defined as the set of alterations introduced upon the therapeutic effect of a given drug stemming from the coadministration of one or more medications. ${ }^{6,7}$ Factors associated with medication-related problems in these patients include: more than three concurrent disease states present; medication regimen changed four or more times during the past 12 months; five or more medications in present drug regimen; history of noncompliance; presence of drugs that require therapeutic monitoring, and presence of diabetes. ${ }^{8}$ Patients with CKD need more care in the center due to multiple medications and investigating drug interactions is the core for providing patient care. For this purpose the current study has designed to investigate drug-drug interaction among $\mathrm{CKD}$ patients in tertiary care hospital.

\section{MATERIALS AND METHODS}

A Hospital based prospective, observational study conducted for a period of 6 months (January to June 2016) in the Medicine Ward of Kempegowda Institute of Medical Science (KIMS) Hospital and Research Center, a tertiary care teaching hospital to investigate drug interaction among chronic kidney disease patients. 
Inclusion Criteria: Patients with $\mathrm{CKD}$ admitted to medicine ward having a creatinine clearance $\leq 50 \mathrm{ml} / \mathrm{min}$ at admission, $\geq$ 18 years and admitted for $>24 \mathrm{~h}$ were included.

Data collection and Documentation: Patients with CKD admitted to medicine ward in KIMS hospital, receiving drug therapy and fulfilled inclusion criteria were included in the study. Medication charts of all patients with chronic kidney disease admitted to the medicine ward during the study period were analysed for drug interactions. Patient's demographic details, drug dose, drug frequency and generic name of all prescribed drugs were documented. The MICROMEDEX Health Series database contains information on medications, etiology, epidemiology, diagnosis and treatment has been used for identifying DDIs. The interactions were classified based on their severity. The potential drug-drug interactions were categorized according to their level of severity using the drug interaction MICROMEDEX Health Series database. Data analysis was performed using the Statistical Package for Social Sciences (SPSS) software program. For the analysis of the data and Microsoft word and Excel have been used to generate tables.

Table 1: Sex Distribution, Relation between Sex, Age Group, Stage of CKD and DDIs

\begin{tabular}{|c|c|c|}
\hline \multicolumn{3}{|c|}{ Sex Distribution (n=108) } \\
\hline Sex & Number & Percentage \\
\hline Male & 68 & 62.96 \\
\hline Female & 40 & 37.04 \\
\hline \multicolumn{3}{|c|}{ Relation between Sex and DDIs } \\
\hline Male & 51 & 47.22 \\
\hline Female & 27 & 25.00 \\
\hline \multicolumn{2}{|c|}{ Relation between age group and DDIs } \\
\hline Age (years) & Number of DDIs & Percentage \\
\hline $18-30$ & 2 & 1.85 \\
\hline $31-50$ & 16 & 14.81 \\
\hline $51-70$ & 33 & 30.56 \\
\hline $71-80$ & 27 & 25.00 \\
\hline$>80$ & 0 & - \\
\hline Relation between Stage of CKD and DDIs \\
\hline Stage 2 & 16 & 14.81 \\
\hline Stage 3 & 25 & 23.15 \\
\hline Stage 4 & 37 & 34.26 \\
\hline \multicolumn{3}{|c|}{} \\
\hline
\end{tabular}

\section{DISCUSSION}

A hospital based prospective, observational study was conducted to identify and determine the drug-drug interactions and to categorize them based on their severity in patients with chronic kidney disease patients admitted to medicine ward in KIMS Hospital \& Research Centre, Bangalore. The prescriptions of 108 patients with CKD were analyzed during the daily ward rounds in the study period and 388 DDIs has been determined in $78(72.2 \%)$ of patients in the period of study and the remaining $30(27.7 \%)$ had no DDIs. Classification of DDIs based on their severity as serious, significant and minor showed that $220(56.7 \%)$ of the DDIs were significant, 111 $(28.61 \%)$ of the DDIs were minor and $57(14.69 \%)$ of the DDIs were serious. Similar study conducted to assess DDIs and their severity showed that majority of the DDIs were of moderate (significant) intensity followed by minor. ${ }^{9}$ The results of present study also showed the DDIs of significant severity are highest in number.On correlating between patient's age group and DDIs it has been found that most number of recorded DDIs, 33 patients $(30.56 \%)$ were belong to age group between $51-70$ years and in

\section{RESULT}

The prescriptions of 108 patients who fulfilled inclusion criteria of study were analyzed during the study period to determine drug-drug interactions (DDIs). Among all included patients, 68 $(62.96 \%)$ male and $40(37.04 \%)$ were female. Out of total patient included in the study, 78 patients medical record showed 388 DDIs during patient's medical record analysis.In correlation between sex and DDIs, $51(47.22 \%)$ patients were male and 27 $(25 \%)$ patients were female.On correlating between patient's age group and DDIs it has been found that most number of recorded DDIs, 33 patients $(30.56 \%)$ were belong to age group between 51-70 years (Table 1). DDIs has been classified into serious, significant and minor. Out of total 388 DDIs, 57 $(14.96 \%), 220(56.7 \%)$ and $111(28.61 \%)$ were serious, significant and minor DDIs respectively.Among all prescribed drugs, diuretics were the drug class with highest number of DDIs followed by antihypertensive agents, hypoglycemia agents and antithrombotic agents respectively. In relationship between polypharmacy and DDIs the highest number of patients, 48 (44.44\%) have been received 13-17 medications (Table 2).

Table 2: DDIs Severity, Drug Classes and Polypharmacy Associate with DDIs

\begin{tabular}{|c|c|c|}
\hline Severity of DI & Number & Percentage \\
\hline Serious & 57 & 14.69 \\
\hline Significant & 220 & 56.70 \\
\hline Minor & 111 & 28.61 \\
\hline \multicolumn{3}{|c|}{ Drug Classes Associated with Drug Interaction } \\
\hline Diuretics & 105 & 27.06 \\
\hline antihypertensive Agents & 89 & 22.94 \\
\hline Hypoglycemic Agents & 78 & 20.10 \\
\hline Antithrombotic Agents & 64 & 16.49 \\
\hline Corticosteroids & 37 & 9.54 \\
\hline Mineral Supplements & 15 & 3.87 \\
\hline \multicolumn{3}{|c|}{$\begin{array}{c}\text { Number of Prescribed Drug Associated with Drug } \\
\text { Interactions }\end{array}$} \\
\hline 3 to 7 & 5 & 4.63 \\
\hline 8 to 12 & 28 & 25.93 \\
\hline 13 to 17 & 48 & 44.44 \\
\hline 18 to 22 & 27 & 25.00 \\
\hline
\end{tabular}

relationship between polypharmacy and DDIs the highest number of patients, $48(44.44 \%)$ have been received $13-17$ medications. Richard M. Goldberg et al undertook a study to determine the potential for adverse drug interactions in a highrisk population and to characterize drug-drug interactions concluded that patients taking three or more medications are at substantial risk for adverse drug-drug interactions. ${ }^{10}$ The observed result in this study also support the fact that polypharmacy is one of the important factors for drug-drug interactions. On correlation between stage of CKD and DDIs, the result showed occurrence of DDIs is high among patient with progressed stage of CKD. Study conducted by Alessandra Batista Marquito with aim to identify potential interactions among drugs prescribed to patients with $\mathrm{CKD}$ on conservative treatment, and factors associated with their occurrence concluded drug associations in individuals with CKD were related to high prevalence of serious DDIs, especially in the later stages of the disease. ${ }^{2}$ Among all prescribed drugs, diuretics were among drugs with highest number of DDIs. A study to assess DDIs also showed that (diuretic) furosemide is a drug with high probability of causing DDIs. ${ }^{11}$ 


\section{Limitation}

Although the study has reached its aims, there were some limitations. First the study requires follow up of DDIs in the patients to look for incidence of actual DDIs and second the number of patient prescriptions analyzed in the study could have been of a higher number.

\section{REFERENCES}

1. Gunar Stemer, Rosa Lemmens-Gruber. Clinical pharmacy activities in chronic kidney disease and end-stage renal disease patients: a systematic literature review.BioMed Central Nephrology 2011; 12(35): 2-12.

2. Alessandra Batista Marquito, Natália Maria da Silva Fernandes, Fernando Antonio Basile Colugnati, RogerioBaumgratz de Paula. Identifying potential drug interactions in chronic kidney disease patients. JornalBrasileiro de Nefrologia2014; 36(1):26-34.

3. Corsonello A, Pedone C, Corica F, Mussi C, Carbonin P, AntonelliIncalzi $\mathrm{R}$ et al.Concealed renal insufficiency and adverse drug reactions in elderly hospitalized patients. Archived Internal Medicine 2005; 165(7):790-5.

4. Gabardi S, Abramson S. Drug dosing in chronic kidney disease. Medical Clinic of North America 2005; 89: 649687.

5. Sathvik BS, Mangasuli S, Narahari MG, Gurudev KC, Parthasarathi G. Medication knowledge of hemodialysis patients and influence of clinical pharmacist provided education on their knowledge. Indian Journal of Pharmaceutical Sciences2007;69:232.
6. Leone R, Magro L, Moretti U, Cutroneo P, Moschini M, Motola D.Identifying adverse drug reactions associated with drug-drug interactions: data mining of a spontaneous reporting database in Italy. Drug Safety 2010; 33(8):667-75.

7. Pirmohamed M. Drug-drug interactions and adverse drug reactions: separating the wheat from the chaff. Wien KlinWochenschr2010; 122(3-4):62-4.

8. Harold J Manley, Debra K Drayer, Richard S Muther. Medication-related problem type and appearance rate in ambulatory hemodialysis patients. BioMed Central Nephrology 2005; 46(4):669-80.

9. Kulkarni V, Bora SS, Sapineni S, Saji M, Siraj S. A Study on Drug-Drug Interactions through Prescription Analysis in a South Indian Teaching Hospital. Therapeutic Advanced Drug Safety. 2013; 4(4).

10. Goldberg RM, Mabee J, Chan L, Wong S. Drug-Drug and Drug-Disease Interactions in the Emergency Department: Analysis of a High-Risk Population. American Journal of Emergency Medicine 1996; 14(5): 447-450.

11. Legese Chelkeba, Fessahaye Alemseged, Worku Bedada. Assessment of potential drug-drug interactions among outpatients receiving cardiovascular medications at Jimma University specialized hospital, South West Ethiopia. International Journal of Basic \& Clinical Pharmacology 2013; 2(2): 144-152.

\section{Cite this article as:}

Yasaman Ghanbari, Kiran Nagaraju. Identifying drug-drug interaction among chronic kidney disease patients in tertiary care hospital. Int. Res. J. Pharm. 2016;7(7):54-56 http://dx.doi.org/10.7897/2230-8407.07785

Disclaimer: IRJP is solely owned by Moksha Publishing House - A non-profit publishing house, dedicated to publish quality research, while every effort has been taken to verify the accuracy of the content published in our Journal. IRJP cannot accept any responsibility or liability for the site content and articles published. The views expressed in articles by our contributing authors are not necessarily those of IRJP editor or editorial board members. 